nutritional management may lead to improved care outcome such as successful ETT removal.

Conclusions Critical care settings should develop a nutritional management protocol for critically ill patients.

\section{ADVERSE CHILDHOOD EXPERIENCES AFFECT PSYCHOLOGICAL OUTCOMES OF INJURY IN URBAN BLACK MEN IN THE US}

${ }^{1}$ Therese S Richmond, ${ }^{1}$ Sara F Jacoby, ${ }^{2}$ Nancy Kassam-Adams, ${ }^{2}$ Justine Shults, ${ }^{1}$ Jessica Webster, ${ }^{1}$ Andrew Robinson, ${ }^{1}$ Patrick Reilly, ${ }^{3}$ John Rich, ${ }^{1}$ Douglas Wiebe. ${ }^{1}$ University of Pennsylvania, USA; ${ }^{2}$ Children's Hospital of Philadelphia, USA; ${ }^{3}$ Drexel University, USA

\subsection{6/injuryprev-2016-042156.782}

Background Injury is not evenly distributed across race and class. In the U.S., urban Black men are at high risk for injury and poor outcomes from injury. Our purpose was to examine the contribution of adverse childhood experiences (ACEs) to post traumatic stress disorder (PTSD) and depression after recent serious physical injury in Black men.

Methods This prospective, cohort follow-up study consecutively enrolled adult Black men hospitalised for serious injury at a Level I Trauma Centre. Men with head injury or currently receiving medical treatment for PTSD or depression, and those in police custody were excluded. ACES (7) were collected by self-report during the intake interview. The outcomes of PTSD (measured by the PCL-C) and depression (measured by the QID-SR 16 ) were collected during in-person interviews in the men's homes 3 months after hospital discharge.

Results 320 (mean age $=36.8$ years) were enrolled. Injury was classified as unintentional $(50 \%)$ or intentional, i.e. the result of interpersonal violence (50\%). The mean number of ACEs did not differ between intentional and unintentional injuries (2.64 vs. 2.35, $\mathrm{p}=0.187) .81 \%$ reported at least $1 \mathrm{ACE}, 45 \%$ reported 3 or more ACEs and 34\% reported $\geq 4$ ACEs. Intentional injuries were associated with higher mean PCL-C scores (42.4 vs. 33.7, p <0.001) and higher mean QID-SR scores (10.0 vs. 7.6, p <0.001). In adjusted multiple regressions, younger age, intentional injury, and number of ACEs were independently associated with higher PCL-C scores. Intentional injury and number of ACEs were independently associated with higher QID-SR scores.

Conclusions This sample of urban Black men reported substantial histories of childhood trauma and adversity. Results provide evidence that adverse childhood experiences increase the risk for depression and PTSD after serious injury. (Funded: NIH R01NR013503 to Dr. Richmond)

\section{IMPACT OF HELMET USE ON TRAUMA CARE OUTCOME IN MOTORCYCLE ACCIDENT PATIENTS OF KHON KAEN HOSPITAL}

Tawatchai Impool, Wittaya Chadbunchachai, Varunchaporn Polkert. Trauma and Critical Care Centre, Khon Kaen Hospital, Thailand

\subsection{6/injuryprev-2016-042156.783}

Background Road Traffic Injury (RTI) is the burden and serious problem, especially in Thailand, it is the major problem. The policy of helmet use was established for many years, but the proportion of helmet use is still unsatisfied. We would like to show that how do the helmet use impact in term of outcome and motivate our patients to use it, as well.

Methods Cross sectional study and data were extracted from trauma registry system of Khon Kaen Hospital (KKH). We enrolled motorcycle accident patients who visited or/and admitted to our hospital between January 2014 to December 2014. We use Chi-square and Fisher exact test for analysis data.

Results Overall we enrolled 2,473 eligible patients. Of these, helmet used patients were 11.85\% (293). Low Glasgow Coma Scale (GCS) [OR (odds ratio), 6.39; 95\%, CI: (confidence interval), 3.63-11.24; $\mathrm{p}<0.0001]$, High Abbreviated Injury Scale (AIS) [OR, 3.63; 95\%, CI: 2.50-5.27; $<<0.0001]$, and mortality rate [OR, 10.06; 95\%, CI: 2.47-40.84; $<<0.0001]$ were associated with non-helmet used patients. Over all mortality rate was $5.78 \%$ Conclusions This study was shown strongly impact of the benefit in helmet used motorcycle accident patients. Obviously, it was able to reduce severity of injury and mortality rate, but there was still low proportion of helmet use in these patients. The helmet use policy should be emphasised and encouraged by the Government more and more.

\section{CARE AT THE RIGHT LEVEL: REFERRALS OF CHILDREN WITH BURN INJURIES AT A LARGE REFERRAL HOSPITAL}

Anders Klingberg, Marie Hasselberg, Tim Stenberg. Karolinska Institutet, Sweden

\subsection{6/injuryprev-2016-042156.784}

Introduction Severe burn injuries require timely medical attention at an appropriate level of care. Correct referral of patients with burns depends on accurate estimation of injury severity. This study aims to determine how often patients at a trauma unit in a large referral hospital in South Africa are referred to the Burn Unit according the guidelines for burn referrals.

Methods Patients presenting with a burn injury to the trauma unit at the Red Cross War Memorial Children's Hospital (RXH) during a 7-months period were included in the study. Patient medical records were studied and compared to the Western Cape Guidelines for referral of patients with burns to determine the number of patients that met the criteria, as well as the number who did not meet the criteria.

Result In total, 702 patients were included in the study. The most common reasons for referral were patients "under two years of age", and "burns to special areas". Out of the 702 patients that were seen at the trauma unit, 499 were transferred to the burns unit. Out of the 499 patients who were referred, $94 \%$ met the criteria for referral. Out of the patients who were not referred to the burns unit, $80 \%$ also met the criteria for referral. This group of patients differed in that they had significantly smaller burns compared to those who were referred.

Conclusion The data suggests that most patients were referred according to the guidelines, with a potential over-triage in very few cases. However, a large numbers of patients met the criteria for referral but were not transferred to the burns unit. These burns were mostly minor burns that likely did not require specialist care. In conclusion, while referral guidelines do catch the patients that need referral, they also catch many that do not need referral. 